\title{
ESTILOS DE APRENDIZAJE Y RENDIMIENTO ACADÉMICO EN ALUMNOS DE UN LICEO EN BUCAREST
}

\section{LEARNING STYLES AND ACADEMIC PERFORMANCE IN STUDENTS OF A HIGH SCHOOL IN BUCHAREST}

\author{
Laurențiu-Cătălin Prună \\ LiceoTeológico Adventista "Ștefan Demetrescu”, Rumania \\ prunicalaur@yahoo.com \\ https://orcid.org/0000-0002-5170-402X
}

\begin{abstract}
RESUMEN
Esta investigación tuvo como objetivo determinar si existe una relación entre los estilos de aprendizaje y las calificaciones de 308 estudiantes de entre 15 y 18 años, de un liceo en Bucarest. Para identificar los estilos de aprendizaje, se utilizó el Cuestionario Honey-Alonso de Estilos de Aprendizaje, el cual está compuesto por 80 items que responden a cuatro estilos de aprendizaje: activo, reflexivo, teórico y pragmático. La prueba estadística utilizada fue el análisis de regresión lineal múltiple. Los resultados mostraron que los estilos de aprendizaje activo y reflexivo son predictores significativos de las calificaciones de los estudiantes. Los estilos teórico y pragmático no contribuyen significativamente a la predicción del rendimiento académico de los estudiantes encuestados. Es importante que los docentes adecuen sus estrategias para que los estudiantes, independientemente de su estilo de aprendizaje, tengan un desempeño exitoso.
\end{abstract}

Palabras clave: estilos de aprendizaje, rendimiento académico, estudiantes de nivel medio

\section{ABSTRACT}

This research aimed to look for a relationship between the learning styles and the grades of 308 students between 15 and 18 years old from a high school in Bucharest. The Honey-Alonso Learning Styles Questionnaire was used to identify the learning styles. It is composed of 80 items that respond to four learning styles: active, reflective, theoretical, and pragmatic. Multiple linear regression analysis was used as a statistical test. The results showed that active and reflective learning styles are significant predictors of student scores. The theoretical and pragmatic styles do not contribute significantly to predicting the academic achievement of the surveyed students. Therefore, it is vital that teachers adapt their strategies so that students, regardless of their learning style, have a successful performance.

Keywords: learning styles, academic achievement, high school students 


\section{Introducción}

Al iniciar los niños el proceso de educación formal, sus expectativas y las de sus padres son altas. No obstante, la realidad es que no todos los estudiantes logran un desempeño que cumpla con ellas. Al parecer, uno de los factores que influye en el rendimiento escolar es el estilo de aprendizaje preferido del alumno (Cutolo y Rochford, 2007; JilardiDamavandi et al., 2011).

El estilo de aprendizaje se define a partir de la base heredada de cada individuo, las experiencias particulares de la vida pasada y las demandas del entorno actual que enfatizan las habilidades de aprendizaje. El estilo de estudio del estudiante, junto con el de su maestro, puede mejorar sus logros académicos. Ambos pueden crear un mejor estudiante (Manochehri, 2008). Por su parte, Kuo-Kuang et al. (2015) afirman que los estilos de aprendizaje se refieren a cómo los estudiantes abordan el proceso de aprendizaje, mientras que Petropol Serb y Petropol Serb (2013) agregan que son elementos de suma importancia, por lo que deben ser variados y adaptados según el grupo educado.

Los investigadores en el campo de la educación presentan diversos modelos de estilos de aprendizaje, así como instrumentos de medida para ayudar a profesores y estudiantes. El conocimiento de los estilos de aprendizaje puede ayudar en el proceso de enseñanza-aprendizaje, para que los resultados académicos tengan un rumbo ascendente. Los estudiantes cuyo estilo de aprendizaje es compatible con el estilo de enseñanza de los profesores tienden a retener la información por más tiempo, aplicarla de manera más efectiva y posteriormente tener una actitud positiva hacia el aprendizaje de esas materias (Dinakar et al., 2005).
Cualquier profesor, independientemente de la estructura académica en la que trabaje, sabe que la forma en que los estudiantes aprenden varía enormemente. Cada alumno llega a clase con determinadas formas de aprendizaje, expectativas y necesidades a las que los profesores deben prestar atención para desarrollar experiencias de aprendizaje (Mupinga et al., 2006). Cada uno de los estudiantes tiene fortalezas que pueden desarrollarse y mejorarse mediante una instrucción eficaz.

Tulbure (2012) señala que el estilo de aprendizaje es un tema complejo, tanto para estudiantes como para profesores. Desde el punto de vista de los estudiantes, el estilo de aprendizaje indica una preferencia general por el aprendizaje e incluye dimensiones cognitivas, afectivas, psicomotoras y fisiológicas. Por otro lado, teniendo en cuenta la perspectiva de los docentes, el hecho de que los estudiantes tengan estilos diferentes es un desafío constante, porque implica diagnosticar estilos de aprendizaje individuales y adaptar la enseñanza a ellos.

Cada individuo tiene un estilo de aprendizaje único, cualidades personales que influyen en la capacidad de acumular información, interactuar con colegas y participar en experiencias de aprendizaje (Mupinga et al., 2006). Cada persona tiene una forma personal de involucrarse en la resolución de una tarea y de relacionarse con determinados temas.

Así, cada niño durante su evolución escolar forma su propio ritmo de aprendizaje. El estilo de aprendizaje es la expresión de un aprendizaje estratégico específico en la actividad de aprendizaje (Afanas, 2014). Los estilos de aprendizaje son el resultado de las preferencias por una forma particular de aprender influenciada por el contexto del entorno de 
aprendizaje y la forma general de conducir de cada alumno. A menudo, el término estilos de aprendizaje se asocia con estilos cognitivos, estilos de pensamiento o formas de aprendizaje (Rassool y Rawaf, 2007).

En general, los profesores enseñan en un estilo acorde con sus preferencias que consideran efectivo para ellos mismos (Healey et al., 2005). Los estudiantes cuyo estilo de aprendizaje es compatible con el estilo de enseñanza de los profesores tienden a retener la información por más tiempo, aplicarla de manera más efectiva y tener una actitud positiva posterior al curso hacia los temas de aprendizaje (Dinakar et al., 2005).

Los estudios de Rosenfeld y Rosenfeld (2004) encontraron que los profesores que eran capaces de describir su estilo de aprendizaje, siguiendo un curso específico de identificación de estilos, también mejoraron su capacidad para comprender a sus alumnos y trabajar de manera diferente.

\section{Estilos de aprendizaje}

Rassool y Rawaf (2007) identificaron cuatro estilos de aprendizaje: activo, reflexivo, teórico y pragmático. Quienes tienen un estilo de aprendizaje predominantemente activo están dominados por experiencias inmediatas y son los primeros en interesarse por lo que está sucediendo aquí y ahora. Les gusta iniciar nuevos desafíos y ser el centro de atención. Los que tienen un estilo reflexivo observan experiencias y prefieren analizarlas en detalle antes de actuar; son buenos oyentes, cautelosos y tienden a pasar inadvertidos. Los teóricos adoptan un enfoque lógico y racional para resolver problemas, pero necesitan un plan con un objetivo claro; les interesa muy poco aprender algo que aparentemente no tiene propósito cuando las actividades no están bien organizadas o son ambiguas. Por último, a los pragmáticos les apasiona probar ideas y técnicas para ver si funcionan en la práctica; son personas prácticas, a las que les gusta tomar decisiones y resolver problemas.

De igual manera, Alonso et al. (1994) afirman que el estilo activo se caracteriza por la creatividad, la innovación, la aventura y la vivacidad. El estudiante con tal estilo de aprendizaje será un líder en su grupo; competitivo, con ganas de aprender y experimentar cosas nuevas. El estilo reflexivo, en cambio, se caracteriza por la paciencia, la atención, la minuciosidad y la prudencia. Un estudiante con un estilo de aprendizaje reflexivo será un buen observador y recolector de datos, un investigador atento y curioso, pero también un buen redactor de informes o declaraciones. El estilo teórico se caracteriza por el orden y la disciplina. El alumno con el estilo de aprendizaje teórico planifica sus actividades, es pensador y perfeccionista; siempre está buscando hipótesis, teorías, explicaciones y sistemas de valores. Es un buen explorador. Finalmente, el estudiante que tiene el estilo pragmático es rápido, decidido, positivo, seguro y aplica lo aprendido.

Nevot (2008) identifica para cada estilo de aprendizaje -activo, reflexivo, teórico y pragmático- los principales obstáculos que pueden encontrar los estudiantes y sugiere algunas soluciones que los profesores pueden abordar para mejorar estos temas sensibles.

Los obstáculos más comunes que pueden afectar el desarrollo de un estilo de aprendizaje activo son los siguientes: miedo al fracaso o miedo a cometer errores, ansiedad, sentirse obligado a hacer lo que no quiere, falta de confianza en sí 
mismo y pensar con demasiada atención en determinadas cosas. Las soluciones propuestas incluyen hacer nuevas actividades, cosas que nunca se han hecho antes, activar la curiosidad, practicar la resolución de problemas en grupos, cambiar en las actividades durante las clases, realizar discusiones, comunicar ideas, solucionar ejercicios mediante técnicas repetitivas, permitir errores y estimular el pensamiento crítico (Nevot, 2008).

En cuanto al estilo reflexivo, entre los principales obstáculos se hallan la insuficiencia de tiempo dedicado a la planificación y al pensamiento, la obligación de cambiar de actividad rápidamente, la impaciencia, el descontrol y la falta de orientación hacia la finalidad. Los alumnos prestan más atención al trabajo en sí que al resultado. Los docentes pueden mejorar esto practicando la escritura con detenimiento, usando pizarrones para realizar determinadas tareas, desarrollando protocolos, recopilando información por medio de la observación, la comunicación oral y la investigación, agregando nueva información, dando tiempo al pensamiento creativo, brindando modelos a seguir, introduciendo una etapa de reflexión en cada acción, despertando la satisfacción de conocer y activando y manteniendo el interés (Nevot, 2008).

Los estudiantes que tienen un estilo de aprendizaje predominantemente teórico pueden enfrentar los siguientes obstáculos: necesidad de ceñirse a las primeras impresiones, preferencia por la intuición y la subjetividad, desagrado por los enfoques estructurados y organizados, dependencia excesiva de otros (profesores o colegas), preferencia por la espontaneidad y el riesgo, incapacidad para convertir pensamientos en acción e incapacidad para completar y realizar el trabajo. Las sugerencias ofrecidas se refieren a la lectura atenta de los teoremas, de los problemas; análisis de situaciones complejas; anticipación para encontrar soluciones y superar los obstáculos; resumen de teorías; formulación de conclusiones; formulación de preguntas; perseverancia; práctica de memorización y automatización; y aplicación de conceptos (Nevot, 2008).

El estilo de aprendizaje pragmático puede plantear ciertos problemas a los estudiantes, tales como pensamiento exagerado sobre cosas útiles, falta de visión sobre la utilidad de lo aprendido, no completar asignaturas, distracción y falta de concentración. Son posibles sugerencias para remediar estos problemas la autocorrección y la autoevaluación, la búsqueda de ayuda de personas experimentadas, los experimentos y las observaciones, el estudio de técnicas utilizadas por otros, los juegos de rol, los ejercicios y el uso de imágenes (Nevot, 2008).

Se ha encontrado que los estilos de aprendizaje están estrechamente relacionados con el rendimiento académico (Wang et al., 2006; Al-Balhan, 2007). La preferencia por un estilo de aprendizaje particular varía según el género (Prajapati et al., 2011), la edad, la experiencia y la madurez (Long y Coldren, 2006; Palloff y Pratt, 2005), el estilo de pensamiento, la estructura del intelecto (Sheridan y Steele-Dadzie, 2005) y la disciplina (Dinakar et al., 2005). El estilo de enseñanza del profesor puede mejorar el estilo de aprendizaje del estudiante (Long y Coldren, 2006). El estilo, la capacidad y la velocidad de aprendizaje difieren de una persona a otra.

Se puede afirmar con certeza que cada alumno tiene su propio estilo de 
aprendizaje. La forma en que adapte los métodos de aprendizaje a su propio estilo determinará la obtención de resultados óptimos y, según las investigaciones, existe una relación entre los estilos de aprendizaje y el rendimiento académico. Esta investigación busca explorar si los estilos de aprendizaje de los alumnos de un liceo en Bucarest son predictores significativos de su rendimiento académico.

\section{Método \\ Tipo de investigación}

Este estudio tuvo un enfoque cuantitativo y transversal, de alcance correlacional.

\section{Participantes}

Participaron del estudio 308 estudiantes de un liceo en Bucarest, Rumania. Los instrumentos se aplicaron a todos los estudiantes de 15 a 18 años en los grados IX a XII.

\section{Instrumentos}

Estilos de aprendizaje. Para identificar los estilos de aprendizaje de los alumnos, se utilizó el Cuestionario Honey-Alonso de Estilos de Aprendizaje (CHAEA), el cual contiene 80 ítems dicotómicos. Se marca 1 si el encuestado está de acuerdo y 0 si está en desacuerdo con la afirmación del ítem. Su validez fue demostrada por Alonso et al. (1994) en una población de 1,371 estudiantes de 25 facultades de la Universidad Autónoma y Politécnica de Madrid y 254 alumnos de la Universidad de Sonora, México. Para probar la validez del instrumento, el autor realizó varias pruebas, tales como análisis de contenido, análisis de ítems, análisis factorial de los 80 ítems, análisis factorial de los 20 ítems para cada estilo de aprendizaje.
Según el análisis de alfa de Cronbach, se obtuvieron los siguientes coeficientes de confiabilidad: estilo activo $(\alpha=.63)$, estilo reflexivo $(\alpha=.73)$, estilo teórico $(\alpha=.66)$ y estilo pragmático $(\alpha=.59)$.

Rendimiento académico. Las calificaciones de los estudiantes se obtuvieron de los requisitos escolares del liceo. Se calculó el promedio general de cada uno de los 308 estudiantes que participaron en este estudio.

\section{Análisis de datos}

Para identificar si los estilos de aprendizaje eran predictores significativos del rendimiento académico, se utilizó el análisis de regresión lineal múltiple.

\section{Resultados \\ Análisis descriptivo \\ Los promedios de las puntuaciones de los estilos son muy equilibrados, des- tacándose un breve predominio de las características del estilo reflexivo (ver Tabla 1).}

\section{Tabla 1}

Estadística descriptiva de estilos de aprendizaje

\begin{tabular}{lcc}
\hline \multicolumn{1}{r}{ Estilo } & $M$ & $D E$ \\
\hline Activo & 12.2 & 3.214 \\
Reflexivo & 14.5 & 3.111 \\
Teórico & 13.5 & 2.727 \\
Pragmático & 13.7 & 2.567 \\
\hline
\end{tabular}

Estilo activo. El estilo de aprendizaje activo obtuvo un promedio de 12.2 $(D E=3.21)$. Las respuestas de los estudiantes encuestados muestran que el enunciado 9, "Trato de ser consciente de lo que pasa aquí y ahora", y el enunciado 26 , "Me siento bien con gente espontánea y divertida", obtuvieron el promedio 
más alto $(M=.95, D E=.209)$. El enunciado con el promedio más bajo fue el 67, "Me resulta inconveniente planificar y predecir cosas" $(M=.28, D E=.449)$.

Se observó que a los estudiantes que tienen un estilo predominantemente activo, y a su vez, calificaciones más altas, no les gusta planificar su tiempo y eventos de su vida. Están deseosos de experimentar cosas nuevas, ser espontáneos y dejarse guiar por la intuición.

Estilo reflexivo. El estilo de aprendizaje reflexivo registra un promedio de $14.5(D E=3.11)$. De las respuestas de los estudiantes encuestados se desprende que el enunciado 28, "Me gusta analizar y pensar cosas", obtuvo el promedio más alto $(M=.92, D E=.274)$. Con el mismo promedio, pero con la desviación estándar de .268 , resultó el enunciado 36, "En las discusiones me gusta observar cómo actúan los demás participantes". El enunciado con el promedio más bajo $(M=.28, D E=.448)$ fue el 58, "Hago muchos borradores antes de hacer la redacción final de un artículo".

Es interesante notar que los estudiantes que tienen un estilo predominantemente reflexivo son los que observan con paciencia las realidades que les rodean, están atentos y solo entonces sacan conclusiones. También son los que, antes de lograr un objetivo académico, recopilan cuidadosamente datos e información para luego redactar un informe o trabajo de investigación.

Estilo teórico. El estilo de aprendizaje teórico registró una media de 13.5 $(D E=2.72)$. Las respuestas de los estudiantes encuestados muestran que el enunciado 54, "Siempre trato de sacar conclusiones e ideas claras", obtuvo el promedio más alto $(M=.92, D E=$ .274). El enunciado con el promedio más bajo $(M=.20, D E=.404)$ fue el 23, "No me gusta involucrarme en el ambiente laboral, prefiero mantener relaciones distantes".

Se observó también que los estudiantes que tienen un estilo predominantemente teórico son ordenados, disciplinados y planifican bien su tiempo para las actividades académicas. Son perfeccionistas y siempre buscan razones y explicaciones para las preguntas que deben responder.

Estilo pragmático. El estilo de aprendizaje predominantemente pragmático obtuvo un promedio de 13.7 $(D E=2.56)$. Las respuestas de los estudiantes interrogados muestran que el enunciado 47, "Suelo optar por formas más prácticas y mejores de hacer las cosas", obtuvo el promedio más alto $(M=.91, D E=.288)$. El enunciado con el promedio más bajo $(M=.12, D E$ $=.329$ ) fue el 62, "Rechazo las ideas originales y espontáneas y no las veo como prácticas".

Finalmente, se observó que los estudiantes que tienen un estilo predominantemente pragmático son muy prácticos, rápidos y decididos en sus actividades académicas; muestran una visión clara y planifican su tiempo para alcanzar resultados positivos. Además, los estudiantes pragmáticos aplican lo aprendido, proporcionando una actividad práctica para cualquier lección teórica.

\section{Análisis de regresión múltiple}

Para confirmar si los estilos de aprendizaje activo, reflexivo, teórico y pragmático son predictores significativos con respecto a las calificaciones de los estudiantes, se aplicó el método de regresión lineal múltiple que dio como resultado que dos de los estilos de aprendizaje son predictores significativos, con 
un coeficiente de determinación de $8.8 \%$ $\left(F_{(2.305)}=15.731, p=.000\right)$. Se trata de los estilos de aprendizaje activo y reflexivo.

El coeficiente estandarizado beta en el caso del estilo de aprendizaje activo es igual a $.229(p=.000)$, mostrando una correlación positiva y, en el caso del estilo de aprendizaje reflexivo, es igual a -.144 ( $p=.012)$, mostrando una correlación negativa.

Se observa una tendencia que muestra que, cuanto mayor puntuación se obtenga en el estilo de aprendizaje activo, mayor será el rendimiento académico y cuanto mayor sea la puntuación en el estilo reflexivo, menor será el rendimiento académico.

\section{Otros hallazgos}

Al aplicar la prueba $t$ para observar las diferencias de medias de rendimiento académico según el género, se observó una diferencia significativa entre los $\operatorname{sexos}\left(t_{(265.126)}=3.528, p=.000\right)$. Los alumnos obtuvieron un promedio menor $(M=8.42)$ que las alumnas $(M=8.74)$. En el estilo de aprendizaje reflexivo, se observó una diferencia significativa entre $\operatorname{sexos}\left(t_{(306)}=2.149, p=.032\right)$, donde la media de los alumnos $(M=14.1)$ fue menor al de las alumnas $(M=14.9)$. En el estilo teórico, igualmente se observó una diferencia significativa entre los se$\operatorname{xos}\left(t_{(268,798)}=2.006, p=.046\right)$, donde los alumnos puntuaron más bajo $(M=$ 13.1) que las alumnas $(M=13.8)$. En el estilo pragmático, igualmente se observó diferencia significativa entre los $\operatorname{sexos}\left(t_{(306)}=2.265, p=.024\right)$, donde los alumnos obtuvieron una puntuación menor $(M=14.1)$ que la de las alumnas $(M=13.4)$.

En síntesis, en todos estos contrastes las alumnas puntuaron mejor.

\section{Discusión}

El análisis estadístico mostró que dos de los estilos de aprendizaje, el activo y el reflexivo, son predictores significativos en una proporción de $8.8 \%$ e influyen en las calificaciones de los estudiantes del estudio.

Un estudio de Gutiérrez Tapias et al. (2011) confirmó que la mayoría de los encuestados tenían un estilo de aprendizaje reflexivo, más visible en las niñas. En la presente investigación, los resultados son diferentes. La preferencia por un determinado estilo de aprendizaje no está determinada por el sexo de los encuestados. Sin embargo, se encontró que, en la institución del estudio, las niñas puntúan más alto que los niños en todos los estilos y que el estilo correspondiente a los promedios anuales más altos es el estilo activo.

La presente investigación llama la atención sobre el hecho de que el estilo de aprendizaje reflexivo conduce a resultados de aprendizaje más bajos. Quizás sería bueno que los maestros de esta escuela adaptaran sus métodos de enseñanza a este estilo de aprendizaje para ayudar a los estudiantes a obtener calificaciones más altas. Los docentes deben adaptar sus métodos para atender especialmente a los estudiantes con el estilo de aprendizaje reflexivo para ver si mejoran sus calificaciones. Los resultados de Carrasco Cifuentes y González Méndez (2018) muestran que una correlación de los estilos de aprendizaje de los estudiantes con los de la enseñanza de los docentes convierte la experiencia educativa en una práctica satisfactoria para docentes y estudiantes.

Los estudiantes también deben poder explicar, desde su propia experiencia, qué es un estilo de aprendizaje y cómo puede ayudar a aumentar el rendimiento académico, ya que los estudios muestran 


\section{ESTILOS DE APRENDIZAJE Y RENDIMIENTO ACADÉMICO}

que los maestros que favorecen esta estrategia también han mejorado en términos de comprender a sus estudiantes y trabajar de manera diferente (Rosenfeld y Rosenfeld, 2004).
En suma, es importante que los docentes adecuen sus estrategias para que los estudiantes, independientemente de su estilo de aprendizaje, tengan éxito en su desempeño académico.

\section{Referencias}

Afanas, A., (2014). Rolul stilurilor de învăţare în activitatea educativă. Studia Universitatis Moldaviae, 9(79), 25-30. http://studiamsu.eu/wp-content/uploads/05.-p.25-30.pdf

Al-Balhan, E. M. (2007). Learning styles in relation to academic performance in middle school mathematics. Domes, 16(1), 42-57. https://doi.org/10.1111/j.1949-3606.2007.tb00064.x

Alonso, C. M., Gallego, D. J. y Honey, P. (1994). Estilos de aprendizaje: ¿Qué son? ¿Cómo se diagnostican? Mensajero.

Carrasco Cifuentes, C. F. y González Méndez, M. A. (2018). Estilos de aprendizaje y rendimiento académico en estudiantes universitarios de psicología en Talca. UCMAULE: Revista Académica de la Universidad Católica del Maule, 54, 107-132. https://doi.org/10.29035/ucmaule.54.107

Cutolo, A. y Rochford, R. A. (2007). An analysis of freshmen learning styles and their relationship to academic achievement. College Quarterly, 10(2), 1-17. http://collegequarterly.ca/2007-vol10-num02spring/cutolo rochford.html

Dinakar, C., Adams, C., Brimer, A. y Silva, M. D. (2005). Learning preferences of caregivers of asthmatic children. Journal of Asthma, 42(8), 683-687. https://doi.org/10.1080/02770900500265157

Gutiérrez Tapias, M., García Cué, J. L., Vivas, M. y Santizo Rincón, J. A. (2011). Estudio comparativo de los estilos de aprendizajes del alumnado que inicia sus estudios universitarios en diversas facultades de Venezuela, México y España. Revista Estilos de Aprendizaje, 4(7), 35-62. http:// revistaestilosdeaprendizaje.com/article/view/921/1629

Healey, M., Kneale, P. y Bradbeer, J. (2005). Learning styles among geography undergraduates: An international comparison. Area, 37(1), 30-42. https://doi.org/10.1111/j.14754762.2005.00600.x

JilardiDamavandi, A., Mahyuddin, R., Elias, H., Daud, S. y Shabani, J. (2011). Academic achievement of students with different learning styles. International Journal of Psychological Studies, 3(2), 186-192. https://doi.org/10.5539/ijps.v3n2p186

Kuo-Kuang, F., Peng-Wei, X. y Chung-Ho Su, M. (2015). The effects of learning styles and meaningful learning on the learning achievement of gamification health education curriculum. Eurasia Journal of Mathematics, Science and Technology Education, 11(5), 1211-1229. https://doi.org/10.12973/eurasia.2015.1413a

Long, H. E. y Coldren, J. T. (2006). Interpersonal influences in large lecture-based classes. College Teaching, 54(2), 237-243. https://doi.org/10.3200/CTCH.54.2.237-243

Manochehri, N. (2008). Individual learning style effects on student satisfaction in a web-based environment. International Journal of Instructional Media, 35(2), 221-228.

Mupinga, D. M., Nora, R. T. y Yaw, D. (2006). The learning styles, expectations, and needs of online students. College Teaching, 54(1), 185-189. https://doi.org/10.3200/CTCH.54.1.185-189

Nevot, A. (2008). Estilos de aprendizaje y enseñanza de las matemáticas. http://www.estilosdeaprendizaje. es/ANevot.pdf

Palloff, R. N. y Pratt, K. (2005). The virtual student: A profile and guide to working with online learners. Jossey-Bass.

Petropol Serb, G. D. y Petropol Serb, I. (2013). Predarea inovantă în inginerie: aspecte ale învățării prin proiect. Buletinul Agir, 18(1), 116-120. https://www.buletinulagir.agir.ro/articol.php?id=1663

Prajapati, B., Dunne, M., Bartlett, H. y Cubbidge, R. (2011). The influence of learning styles, enrollment status and gender on academic performance of optometry undergraduates. Ophthalmic and Physiological Optics, 31(1), 69-78. https://doi.org/10.1111/j.14751313.2010.00798.x

Rassool, G. H. y Rawaf, S. (2007). Learning style preferences of undergraduate nursing students. Nursing Standard, 21(32), 35-41. https://doi.org/10.7748/ns2007.04.21.32.35.c4495

Rosenfeld, M. y Rosenfeld, S. (2004). Developing teacher sensitivity to individual learning differences. Educational Psychology, 24(4), 465-486. https://doi.org/10.1080/0144341042000228852

Sheridan, M. J. y Steele-Dadzie, T. E. (2005). Structure of intellect and learning style of incarcerated youth assessment: A means to providing a continuum of educational service in juvenile justice. The Journal of Correctional Education, 56(4), 347-371. https://www.jstor.org/stable/23282626?seq=1 


\section{CĂTĂLIN PRUNĂ}

Tulbure, C. (2012). Investigating the relationships between teaching strategies and learning styles in higher education. Acta Didactica Napocensia, 5(1), 65-74. http://dppd.ubbcluj.ro/adn/article 5 1 7.pdf

Wang, K. H., Wang, T. H., Wang, W. L. y Huang, S. C. (2006). Learning styles and formative assessment strategy: Enhancing student achievement in web-based learning. Journal of Computer Assisted Learning, 22(3), 207-217. https://doi.org/10.1111/j.13652729.2006.00166.x

Recibido: 18 de agosto de 2020

Revisado:11 de marzo de 2021 Aceptado: 15 de junio de 2021 